accurate modelling at high frequencies, as a lumped-element approximation of the frequency response of the device is not necessary.

\section{F. FILICORI}

10th December 1990

Dipartimento di Elettronica, Informatica e Sistemistica

Università di Bologna, Viale Risorgimento 2-40136 Bologna, Italy

\section{References}

1 HaIGH, D., and EVERARD, J. (Eds.): 'GaAs technology and its impact on circuits and systems' (Peter Peregrinus Ltd. on behalf of IEE, 1989)

2 RAUSCHER, C., and WILLING, H.: 'Simulation of nonlinear microwave FET performance using a quasi-static model', IEEE Trans., 1979, MTT-27, pp. 834-840

3 Filicori, F., and monaco, v. A.: 'Computer-aided design of nonlinear microwave circuits', Alta Frequenza, 1988, LVII, (7), pp. $355-378$

4 GHIONE G., FILICORI, $F$, and NALDI, C.: 'Physical modeling of GaAs MESFET's in an integrated CAD environment: from device technology to microwave circuit performance', IEEE Trans., 1989, MTT-37, pp. 457-468

5 SANGO, M., et al.: 'A GaAs MESFET large-signal circuit model for non-linear analysis'. IEEE MTT-S digest, 1988, pp. 1053-1056

\section{ECG DATA COMPRESSION BY SUB-BAND CODING}

Indexing terms: Data compression, Coding, Biomedical electronics

A data compression technique is presented for discrete-time electrocardiogram signals. The single lead electrocardiogram signal is decomposed into several multiresolution subsignals by using a quadrature mirror filter bank. The resultant subsignals are compressed according to their frequency contents using various coding methods, including a discrete cosine transform based technique and pulse code modulation with variable length coding. Compression ratios as high as 5.7 are obtained without introducing any visual distortion.

Introduction: Computerised electrocardiogram (ECG) processing systems have been widely used in ECG analysis. ${ }^{1,2}$ The discrete-time ECG compression has become a necessity for many reasons. These include reducing the memory space in ECG data bases, reducing the transmission period of real time ECGs over telephone networks and increasing the recording time of the ambulatory devices.

Current data compression techniques for ECG signals can be classified as predictive coding (e.g. DPCM) methods and transform coding methods (see Reference 3 for an extensive survey of these methods). We present a sub-band coding based compression scheme for ECG signals. The ECG signal is split into subsignals by using a quadrature mirror filter bank in a tree structured fashion.

Sub-band coding has been successfully applied to speech and image coding. 5,6 In the coding of sub-band signals, advantage is taken of the nonuniform distribution of energy in the frequency domain to judiciously allocate the bits to represent the sub-band signals. In our method the subsignal with the lowest frequency content is encoded by using a discrete cosine transform (DCT) based compression scheme ${ }^{7}$ and the other subsignals are quantised using deadzone quantisers. The resulting data is coded using runlength coding of zero valued samples and variable length coding of the nonzero samples.

Description of procedure: Quadrature mirror filter bank (QMFB) is one of the building blocks used in multirate signal processing. It finds applications in situations where a discretetime signal is to be split into a number of consecutive bands in the frequency domain. This division into frequency components removes the redundancy in the input and has the advantage that the number of bits used to encode each frequency band can be different, so that the encoding accuracy is always maintained at the required frequency bands.

The whole structure that is used to compress ECG signals is shown in Fig. 1. The single lead ECG signal is decomposed into four subsignals by using a QMF bank in a tree structured fashion and resulting stages of the tree are decimated by a factor of two. In the absence of quantisation errors, a $Q M$ bank based tree structure provides perfect reconstruction, i.e.

$$
y(n)=x(n-K)
$$

$K$ an integer

where $y(n)$ is the output and $x(n)$ is the input of the QMFB. This is because of the fact ${ }^{8}$ that the lowpass, $H_{l}(\omega)$, and the high pass filter, $H_{h}(\omega)$ satisfy the following condition:

$$
\left|H_{l}(\omega)\right|^{2}+\left|H_{h}(\omega)\right|^{2}=1
$$

During the preprocessing of DI lead ECG data sampled at $500 \mathrm{~Hz}$ it is observed that the energy of the ECG signals is highly concentrated at frequencies less than $62.5 \mathrm{~Hz}$. Hence, at least a four frequency band filter bank is necessary. Each subband is then encoded according to criteria that are specific to that band. ECG waveforms depict that all the three bands except the lowest frequency band have noise-like variations, therefore they are coded using uniform quantisers. After quantisation, a code assignment procedure is realised using amplitude and runlength lookup tables which are derived by variable length coding from the histograms of quantised subband signals.

Transform coding was used in Reference 9 to compress discrete-time ECG signals. In our method we use a discrete cosine transform (DCT) based transform coding method to compress the sub-band signals with lowest frequency content It is observed that the ECG signal energy is mainly concentrated in the lowest frequency band. Because of this, the

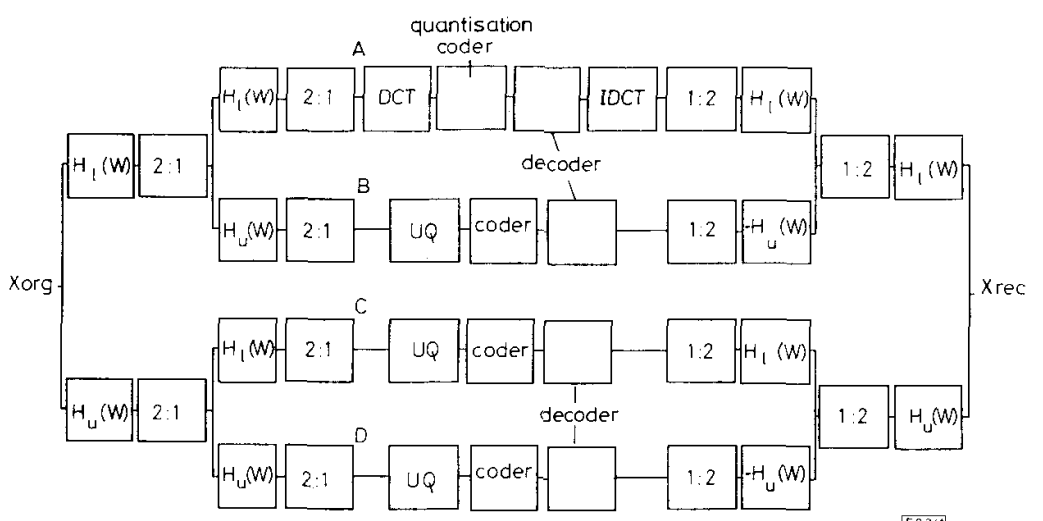

Fig. 1 Four branches sub-band coder structure 
lowband signal has to be carefully coded. The high correlation among neighbouring samples makes the lowband signal a good candidate for efficient predictive or transform coding. We have chosen to use DCT in view of the known efficiency of transform codes.

The DCT of a data sequence $x(n), n=0,1, \ldots,(N-1)$ is defined as

$G(0)=\frac{\sqrt{ }(2)}{N} \sum_{n=0}^{N-1} x(n)$

$G(k)=\frac{2}{N} \sum_{n=0}^{N-1} x(n) \cos \frac{(2 n+1) k \pi}{2 N} \quad k=1,2, \ldots,(N-1)$

where $G(k)$ is the $k$ th DCT coefficient. The inverse discrete cosine transform (IDCT) of $G(k)$ is given as

$$
\begin{aligned}
x(n)=\frac{1}{\sqrt{(2)}} G(0)+\sum_{k=1}^{N-1} G(k) \cos \frac{(2 n+1) k \pi}{2 N} & \\
n & =0,1,2, \ldots,(N-1)
\end{aligned}
$$

After the application of discrete cosine transformation with a block size of $N=64$ samples to the upper branch, the following process is the quantisation of transform coefficients. Because the resulting error is very sensitive to quantisation, only a floating point to integer conversion is applied. The resulting nonzero and zero valued coefficients are coded by amplitude and runlength lookup tables, respectively. The bit streams which are obtained from the coding of four sub-bands are multiplexed and stored.

A fidelity measure called percent root mean square difference (PRD) is employed. ${ }^{3}$ The PRD is defined as

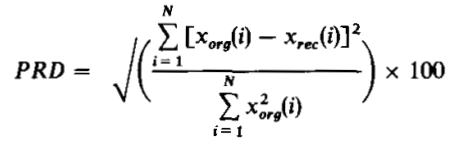

where $x_{\text {org }}$ and $x_{\text {rec }}$ are original and reconstructed data sequences, respectively.

Simulation results: The algorithm given in Fig. 1 is implemented. ECG signals are sampled at $500 \mathrm{~Hz}$ with 12 bit resolution and partitioned into blocks with lengths of 1024 samples each. A 32-tap finite impulse response OMF pair which is described in Reference 8 is used as lowpass, $H_{l}$ and high pass, $H_{h}$ filters. These FIR filters approximate the perfect reconstruction condition (eqn. 2) and also satisfy the following bounds:

$$
0.9943 \leq\left|H_{n}(\omega)\right|^{2}+\left|H_{h}(\omega)\right|^{2} \leq 1.0058
$$

Discrete cosine transforms with block sizes of 64 samples, applied to branches $A$ and $B, C$, and $D$, are coded employing quantisers of seven, three and three levels, respectively. As reported in Table 1, the compression ratio (CR) is determined as 4.6 and the $P R D$ is $3.2 \%$. Also as high a compression ratio as 5.7 was obtained with a $P R D=7.0 \%$ and we observed that it is virtually impossible to distinguish the original and the reconstructed signal as shown in Fig. 2.

Table 1 COMPRESSION RATIOS AND PRD AND MSE VALUES FOR DIFFERENT SCHEMES

\begin{tabular}{lccr}
\hline \multicolumn{1}{c}{ Method } & CR & PRD & MSE \\
\hline & & $\%$ & \\
DCT & $4 \cdot 2$ & $4 \cdot 3$ & $12 \cdot 6$ \\
DCT & $4 \cdot 6$ & $4 \cdot 8$ & $15 \cdot 9$ \\
SBC + DCT & $4 \cdot 2$ & $2 \cdot 6$ & $5 \cdot 1$ \\
SBC + DCT & $4 \cdot 6$ & $3 \cdot 2$ & $8 \cdot 0$ \\
DCT, filtered data & $4 \cdot 5$ & $2 \cdot 1$ & $3 \cdot 1$ \\
DCT, filtered data & $5 \cdot 3$ & $2 \cdot 9$ & $5 \cdot 9$ \\
SBC + DCT, filtered data & $4 \cdot 5$ & $1 \cdot 7$ & $2 \cdot 3$ \\
SBC + DCT, filtered data & $5 \cdot 3$ & $2 \cdot 6$ & $5 \cdot 4$ \\
\hline
\end{tabular}

360
We also applied the DCT coding method to the input ECG signal without performing any sub-band decomposition, e.g. for a $C R$ of 4.6 , the $P R D$ is determined as $4.8 \%$, whereas the sub-band coding method produces a $P R D$ of $3.2 \%$ for the same CR.

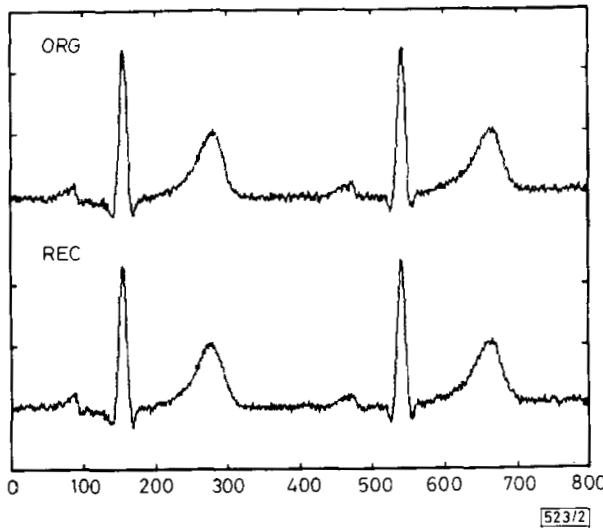

Fig. 2 Original and reconstructed ECG signals with $C R=5.7$ $P R D=7 \cdot 0 \%$

High frequency branches have noise-like variations. It would be reasonable to increase this low correlation to obtain higher compression ratios using a prefilter at the input of the sub-band coder. A lowpass filter with a cutoff frequency equal to $125 \mathrm{~Hz}$ is used and a CR and PRD of 5.3 and $2.9 \%$ are obtained, respectively.

Simulations have been completed on a personal computer and it is seen that the new procedure works 2.5 times faster than the conventional DCT based compression technique. Also, the structure is very convenient for decreasing the execution period using digital signal processors which have special macros for the simplification of the filtering and FFT algorithms.

Conclusion: We presented a new method to compress ECG signals. It is observed that the use of sub-band decomposition based methods produce better compression ratios than transform coding methods. Further work is in progress to employ multidimensional sub-band coding schemes to code multichannel ECG signals.

M. C. AYDIN

6th November 1990

A. E. ÇETIN

Department of

Bilkent Universit

Bilkent, Ankara 06533, Turkey

\section{References}

1 roUNG, $T_{n}$ and HUGGINS, $w$.: 'The intrinsic component theory of electrocardiograpby', IRE Trans, 1962, BME-9, pp. 214-22

2 İDER, Y. Z., and KöYMEN, H.: 'A new technique for line interference IDER, Y. Z., and KöYMEN, H.: 'A new technique for line interference
monitoring and reduction in biopotential amplifiers', IEEE Trans. monitoring and reduction in biop
$1990, \mathrm{BME}-37,(6)$, pp. 624-631

3 Jalaleddine, S. M. S., hUtChens, C. G., STRattan, R. D., and CORERLY, W. A.: 'ECG data compression techniques-A unified approach', IEEE Trans., 1990, BME-37, (4), pp. 329-343

4 ESTEBAN, D., and GALAND, C.: 'Application of quadrature mirror filters to split band voice coding schemes'. Proc. ICASSP, 1977, pp. 191-195

5 wOons, J. W., and O'NEILL, S. D.: 'Sub-band coding of images', IEEE Trans., 1988, ASSP-34, pp. 1278-1288

6 ANSARI, R., ÇETN, A. E., and LEE, S. H.: 'Sub-band coding of images using nonrectangular filter banks'. Applications of Digital Image Processing XI, SPIE, 1988, 974, pp. 315-323

7 AHMED, N., Natarajan, T., and RaO, $\mathrm{K}$. R.: 'Discrete cosine transform', IEEE Trans., 1974, C-23, pp. 90-93

8 CROCHIERE, R. E.: 'Sub-band coding', Bell Syst. Tech. J., 1981, 60 , (7), pp. 1633-1653

9 DYER, S. A., AHMED, N., and HUMMELS, D. R.: 'Vectorcardiographic data compression via Walsh and cosine transforms', IEEE Trans. 1985, EMC-27, (1), pp. 24-34

ELECTRONICS LETTERS 14th February 1991 Vol. 27 No. 4 